\section{SAT0100 FENOFIBRATE REDUCES SERUM URATE IN PATIENTS WITH HYPERURICAEMIA ESTABLISHED ON ALLOPURINOL}

${ }^{1} \mathrm{AL}$ Hepburn, ${ }^{1} \mathrm{MB}$ Hogarth, ${ }^{2} \mathrm{SG}$ Ball, ${ }^{1} \mathrm{SA}$ Kaye, ${ }^{3} \mathrm{MD}$ Feher. ${ }^{1}$ Department of Rheumatology; ${ }^{2}$ Department of Clinical Chemistry; ${ }^{3}$ Lipid Clinic, Chelsea \& Westminster Hospital, London, UK

\subsection{6/annrheumdis-2001.483}

Background Fenofibrate is an established treatment for various forms of hyperlipidaemia and is unique amongst the fibric acid derivatives due to its ability to lower serum urate. This uratelowering effect has not, however, been assessed in patients established on allopurinol, the most widely used drug in the prevention of gout.

Objectives To assess the short-term urate lowering effect of fenofibrate in patients with hyperuricaemia and gout already treated with allopurinol.

Methods 11 patients (10M:1F), mean age 56.5 (range 38-74) with a history of either chronic tophaceous or interval gout in association with hyperuricaemia were assessed in an open crossover study. Each had been treated with allopurinol $300 \mathrm{mg} /$ day or more for at least 3 months and had been free of acute gout for a minimum of 1 month. Each patient received micronised fenofibrate $200 \mathrm{mg}$ once daily for 3 weeks. Before this was commenced, the following were measured: serum urate and creatinine, $24 \mathrm{hr}$ urinary output of urate and creatinine, liver function tests, creatine kinase and fasting serum lipids These were repeated at the end of fenofibrate therapy and again 3 weeks later. Allopurinol was continued throughout the study period.

Results Fenofibrate was associated with a $24 \%$ reduction in serum urate after 3 weeks of treatment (mean $0.36 \pm 0.11$ vs $0.29 \pm 0.07 \mathrm{mmol} / \mathrm{l} ; \mathrm{p}=0.002$ ). The urate-lowering effect was rapidly reversed on its withdrawal $(0.29 \pm 0.07$ vs $0.37 \pm 0.09$ $\mathrm{mmol} / \mathrm{l})$. There was a rise in urate clearance of $56 \%(6.7 \pm 3.1$ vs $10.5 \pm 5.6 \mathrm{ml} / \mathrm{min}$ NR $6-11 ; \mathrm{p}=0.005)$ without any significant change in creatinine clearance. The largest reductions in serum urate occurred in patients with the highest levels at baseline. Both total cholesterol and triglyceride levels in the serum fell, but this was not statistically significant in either case $(5.9 \pm$ 1.0 vs $5.3 \pm 0.9 \mathrm{mmol} / \mathrm{l} ; \mathrm{p}=0.16$ and $2.34 \pm 1.11$ vs $1.77 \pm$ $0.66 \mathrm{mmol} / \mathrm{l} ; \mathrm{p}=0.15$ respectively). Alkaline phosphatase activity was reduced in each patient, confirming compliance with fenofibrate $(70 \pm 15$ vs $53 \pm 12 \mathrm{U} / \mathrm{l})$. Importantly, none of the group developed acute arthritis whilst taking fenofibrate, and the three who continue to take the drug remain free of gouty attacks.

Conclusion Fenofibrate rapidly lowers serum urate by a significant degree in patients with hyperuricaemia who are already established on allopurinol prophylaxis. No adverse effects were seen, in particular a flare of gout. Fenofibrate is a potential novel treatment for hyperuricaemia and the prevention of gout. In particular it may have a useful dual role in patients with coexisting hyperuricaemia and hyperlipidaemia.

\section{REFERENCE}

1 Bastow MD, et al. Hypertriglyceridaemia \& hyperuricaemia: effects of two fibric acid derivatives (bezafibrate \& fenofibrate) in a double-blind, placebo-controlled trial. Metabolism 1988;37:217-20

\section{SAT0101 ANALYSIS OF THE EFFICACY OF CYCLOSPORIN A IN 56 PSORIATIC PATIENTS}

II Krajnc, ${ }^{2} \mathrm{~K}$ Lukanoviè, 'I Holc. 'Department of Rheumatology and Immunology, Internal Clinic Maribor, University Ljubljana, Maribor; ${ }^{2}$ Novartis, Ljubljana, Slovenia

10.1136/annrheumdis-2001.484

\section{Background}

Objectives $20 \%$ of the patients with psoriatic arthritis (PA) may develop a very severe form of arthritis. When the skin disease is extensive, it makes sense to use medications which are thought to be effective for both, the skin and joint disease. The efficacy of the cyclosporin $\mathrm{A}(\mathrm{CyA})$ on the skin lesiones in psoriasis has been shown in several controlled studies. A few authors demonstrated the anti-inflammatory efficacy of the drug in the PA. The most important action of CyA is the inhibition of production of cytokines by T-cells. We have evaluated efficacy and tolerability of CyA in PA patients.56 patients with average age of 47 (range 33-71), 21 female and 35 male have been treated with $3 \mathrm{mg} / \mathrm{kg}$ per day for 6 months. 19 patients received low doses of corticosteroids, nobody received other DMARD during the treatment. Methods Several clinical and laboratory parameters for the assessment of joint status at the start and at the end of the study were employed: Ritchie index, duration in minutes of morning stiffness with a semi-quantitive evaluation the presence or absence of the onychopathy. We also performed at monthly interval the systolic blood pressure values and the laboratory parameters: erythrocyte sedimentation rate, CRP, serum creatinine and blood urea and liver enzymes. We measured a concentration of the drug in serum with Fluorescence polarisation immuno assay (ABOTT) method at the beginning and at the end of the study.

Results The results are shown in the following Table 1. (statistical-analysis t-student-test)

\begin{tabular}{ll} 
Abstract SAT0101 Table 1 & \\
\hline Ritchie index & $\mathrm{p}<0,01$ \\
Morning joint stiffines & $\mathrm{p}<0,05$ \\
ESR & $\mathrm{p}>0,05$ \\
CRP & $\mathrm{p}>0,05$ \\
\hline
\end{tabular}

Conclusion Six of our patients drop out because of high blood pressure, all other patients finished the study. Our results show a good tolerability and efficacy on the joint pain, on the morning stiffness and on the skin lesions.

\section{SAT0102 BONE AND JOINT PROBLEMS IN HAEMODIALYSIS PATIENTS}

GG Demirel, M Yelkovan, H Yılmaz, B Görçin. Physical Medicine and Rehabilitation Centre, Ist. Phys. Medicine and Rehabilitation Centre, Istanbul, Turkey

10.1136/annrheumdis-2001.485

Background Patient receiving maintenance haemodialysis suffer from various problems of the joints, soft tissues and tendons. These effects include avascular necrosis, osseous abnormalities, ligamentous laxity, destructive arthropathies and soft tissue calcifications. IN end stage renal disease, average bone mineral density has been reported modestly reduced. 
Objectives To estabilish the incidence and nature of osteoarticular disease in patients receiving long term treatment with haemodialysis we surveyed all of our patients who had received dialysis.

Methods The subjects were 123 patients who were dialysed for one year to 22 years. We also analysed clinical factors such as age, haemodialysis duration, underlying disorder of chronic renal insufficiency, haemotologic parameters. All patients were seen at an extraclinic attendance when they were asked to evaluate pain and stiffness in each joint. A diagnosis of dialysis associated arthropathy was made in patients by three investigators, using as criteria single or combined presence of carpal tunnel syndrome, erosions and bone cysts of the joints and destructive spondylarthropathy. Plasma üre, creatinin, haemoglobin, ferritin, calcium, phosphorous, ürate, paratthyroid hormon concentrations, alkaline phospatase, magnesium, aliminium, B2 microglobulin, osteocalcin, vit $\mathrm{D}$, hepatit markers were measured before dialysis in patients. Radiographs of the hands were obtained in all patients, in some patients painful joints were also axamined by radiography. We used dual-energy X-Ray absorbtiometry to detect changes in bone mineral density.

Results We found carpal tunnel syndrome in 29 (23.8\%) patients, spondylarthropathy in14 (11.5\%) patients, skeletal deformities in $29(23.8 \%)$ patients, tensynovitis in $6(4.8)$ patients, periarthritis in $10(8.2 \%)$ patients, periarthritis in 10 $(8.2 \%)$ patients and bone cysts in $26(20.7 \%)$ patients. When we compared groups according to durationof hemodialysis treatment; carpal tunnel syndrome, bone cysts and periarthritis were found more frequently in patients receiving hemodialysis treatment for longer time. Bone mineral density was reduced in dialysis patients of both sexes in comparison with the healty subjects. There was significant correlation between bone mineral density and the mean serum B2 microglobulin, magnesium, cretinin and ferritin, alkaline phosphatase levels. Strong relationship was found out among hemodialysis duration and osteoporosis. Depression was observed $66.3 \%$ patients.

Conclusion Changes of the locomotor apparatus in prolonged hemodialysis treatment determine the quality of life with all its consequences for the patient.

\section{REFERENCES}

1 Levenson JL, Glocheski S. Psychological factors affecting end-stage renal disease. Psychosomatics 1993:32,382-8

2 Ferrari AJL, Rothfuss S, Schumacher HR. Dialysis arthropathy; identification and evaluation of a subset of patients with unexplained inflammatory effusions. I Rheumatol. 1997:24(9):1780-6

3 Loevner LA, Adler RS, Martel W. Dialysis related arthropathy in patients on long term hemodialysis: radiographic features. J Clin Rheumatol.1995;1:81-9

\section{SAT0103 ACROMEGALIC ARTHROPATHY: AN ITALIAN STUDY IN 20 PATIENTS}

${ }^{1} \mathrm{~F}$ Schiavon, ${ }^{1} \mathrm{H}$ Marotta, ${ }^{2} \mathrm{P}$ Maffei, ${ }^{2} \mathrm{C}$ Martini, ${ }^{2} \mathrm{C}$ Menegazzo, ${ }^{2} \mathrm{~N}$ Sicolo, ${ }^{3} \mathrm{R}$ Ragazzi, ${ }^{1} \mathrm{~S}$ Todesco. 'Division of Rheumatology, Department of Medical and Surgical Sciences, University of Padova; ${ }^{2}$ Third Medical Clinic, Department of Medical and Surgical Sciences, University of Padova, Padova, Italy; ${ }^{3}$ First Radiology Unit

\subsection{6/annrheumdis-2001.486}

Background Acromegaly is frequently complicated by axial and peripheral joint abnormalities. Although usually mild, severe handicap is not infrequent. In addition soft tissue, cartilage and bony proliferation make acromegaly a peculiar model of noninflammatory joint disease, which is reflected by clinical and radiological findings.
Objectives The aim of this study was to compare the clinical and radiographic features of Italian acromegalic patients with the well- known literature studies.

Methods We studied 20 patients (10 men and 10 women) with acromegaly, admitted to our Department. The diagnosis of acromegaly and the status of the disease were based on clinical, hormonal and radiological features. The duration of disease was assumed as the interval between the clinical onset and the time of the diagnosis and was $5.43 \pm 2.76$ year for men and $8.50 \pm$ 5.66 year for women. Physical examination of peripheral and axial joints and radiological assessment of hands, wrists, shoulders, spine, pelvis hips and knee was performed. Moreover a ultrasonographyc shoulder's study in 8 patients was carried out. All the patients were submitted to a global self assessment of disease status or activity with WOMAC.

Results We found 70\% of acromegalic gave a history of peripheral joint pain, often mild and intermittent and 65\% described spine involvement. At the examination 12 patients described spine pain, mostly mild and poorly localised, 9 shoulder pain with severely disabling symptoms, 4 mild localised knee joint pain, 2 hip pain. Only 2 patients reported hand pain. Over $50 \%$ of patients presented joint hypermobility. We are able to find a correlation between the severity of peripheral joint or spinal abnormalities, WOMAC physical function and stiffness scale score and activity of acromegaly (GH and IGF-1 blood levels) (p $<0.001$ ). In our patients the shoulder represents one of the most painful and disabling joint. Radiographic abnormalities included narrowing of glenohumeral joint, beak-like osteophytes and, in 4 patients, a destructive arthropathy with oval deformation of humeral head. Ultrasonographic studies were carried out in 8 patients and revealed tendons involvement in all the patients, even without radiological abnormalities. We found supraspinatus tendon tear in 3 patients, rotator cuff and bicipital tendinitis in 7 patients and subacromial bursitis in 2 patients.

Conclusion

\section{SAT0104 ARE COAGULOPATHIES INVOLVED IN NONTRAUMATIC AVASCULAR NECROSIS?}

${ }^{1} \mathrm{~N}$ Mehsen, ${ }^{2} \mathrm{JP}$ Vernhes, ${ }^{3} \mathrm{~V}$ Guérin, ${ }^{1} \mathrm{P}$ Germain, ${ }^{1} \mathrm{~L}$ Malterre, ${ }^{1} \mathrm{~J}$ Dehais, ${ }^{3} \mathrm{~F}$ Parrot, ${ }^{1} \mathrm{~T}$ Schaeverbeke. ${ }^{1}$ Rheumatology; ${ }^{2}$ Rheumatology, $\mathrm{CH}$ Libourne, Libourne, France; ${ }^{3}$ Hematology, CHU Bordeaux, Bordeaux

\subsection{6/annrheumdis-2001.487}

Background Various conditions are considered as classical etiologies of nontraumatic avascular necrosis (AVN), such as endogenous or exogenous hypercorticism, alcoholism, barotraumatisms, systemic lupus, diabetes mellitus and hyperlipidemia. However, in many cases, none of these conditions are identified and AVN is considered to be idiopathic.

Objectives To seek thrombophilic disorders in a cohort of patients with AVN.

Methods All patients with AVN hospitalised in our departments during a couple of years were enrolled in this prospective study. Diagnosis of AVN was performed on X-rays and/or MRI when necessary and possible. Biological investigations included: glycemia, cortisolemia, ferritinemia, cholesterolemia, lipoproteinemia, haemoglobin electrophoresis, ANA, antiphospholipid antibodies, anti-B2GP1 antibodies, lupus anticoagulant, antiprothrombin antibodies, antithrombin, protein $\mathrm{C}$, protein $\mathrm{S}$, factor VIII, IX, $\mathrm{XI}$, activated protein $\mathrm{C}$ resistance, prothrombin gene mutation, homocysteinemia and methionin load test. 